\title{
A comparison of imaging modalities for the diagnosis of osteomyelitis
}

Brandon J. Smith ${ }^{1}$, Grant S. Buchanan ${ }^{2}$, Franklin D. Shuler ${ }^{2}$

Author Affiliations:

1. Joan C Edwards School of Medicine, Marshall University, Huntington, West Virginia

2. Marshall University

The authors have no financial disclosures to declare and no conflicts of interest to report.

Corresponding Author:

Brandon J. Smith

Marshall University Joan C. Edwards School of Medicine

Huntington, West Virginia

Email: smith1957@live.marshall.edu 


\section{Abstract}

Osteomyelitis is an increasingly common pathology that often poses a diagnostic challenge to clinicians. Accurate and timely diagnosis is critical to preventing complications that can result in the loss of life or limb. In addition to history, physical exam, and laboratory studies, diagnostic imaging plays an essential role in the diagnostic process. This narrative review article discusses various imaging modalities employed to diagnose osteomyelitis: plain films, computed tomography (CT), magnetic resonance imaging (MRI), ultrasound, bone scintigraphy, and positron emission tomography (PET). Articles were obtained from PubMed and screened for relevance to the topic of diagnostic imaging for osteomyelitis. The authors conclude that plain films are an appropriate first step, as they may reveal osteolytic changes and can help rule out alternative pathology. MRI is often the most appropriate second study, as it is highly sensitive and can detect bone marrow changes within days of an infection. Other studies such as CT, ultrasound, and bone scintigraphy may be useful in patients who cannot undergo MRI. CT is useful for identifying necrotic bone in chronic infections. Ultrasound may be useful in children or those with sickle-cell disease. Bone scintigraphy is particularly useful for vertebral osteomyelitis. Finally, PET scan has demonstrated high sensitivity and specificity; however, its clinical application is limited by its high cost and poor availability. When used appropriately, diagnostic imaging can provide high sensitivity and specificity for detecting osteomyelitis, making radiographic evaluation a crucial step in the diagnostic process of this debilitating condition.

\section{Keywords}

Diagnostic Imaging, Osteomyelitis, Radiology, X-ray, CT, MRI, Bone Scan, Ultrasound

\section{Introduction}

Osteomyelitis is the general term for an infection of bone. The incidence of osteomyelitis has increased over the past several decades, especially in patients greater than 60 years of age. ${ }^{1}$ This increase is believed to have resulted from an increase in diabetes related infections among adults. ${ }^{1}$ In addition to diabetes, a number of other factors can lead to osteomyelitis: bacteremia, open wounds, trauma, surgery, and prosthetic devices. Taking these sources into account, osteomyelitis can be classified into three broad etiologic categories: hematogenous (bacteremia), contiguous spread without vascular insufficiency (trauma, surgery, foreign bodies), or contiguous spread with vascular insufficiency (chronic wounds, diabetic foot infections). ${ }^{2}$ Osteomyelitis can be further subdivided into acute and chronic based on histological findings rather than duration of symptoms; acute infection demonstrates inflammatory bone changes whereas chronic infection reveals necrotic bone. ${ }^{3}$ Imaging plays a vital role in the diagnosis of osteomyelitis. This review describes the role of common imaging modalities utilized in clinical practice based on current literature.

\section{Methods}


NCBI's PubMed database was utilized to search for literature pertaining to the diagnostic imaging of osteomyelitis. Combinations of the following search terms were used: bone infection, osteomyelitis, diagnosis, radiology, diagnostic imaging, imaging, magnetic resonance imaging, MRI, computed tomography, CT, x-ray, plain film, radiograph, bone scan, bone scintigraphy, positron emission tomography, PET, ultrasound. Primary and secondary sources were screened for relevance by title and the contents of the abstract. Potential sources were then downloaded and their contents were scrutinized for relevance. The authors also incorporated guidelines from the Infectious Diseases Society of America (IDSA). A total of 23 references were included in the final review.

\section{Presentation}

Patients with osteomyelitis can experience a variety of symptoms depending on the underlying etiology of the infection. Common but non-specific symptoms can include fever, chills, fatigue, lethargy, or irritability. Localized symptoms may include pain, erythema, or swelling over the affected bone. ${ }^{4}$ A thorough history and physical exam should be performed on each patient suspected of having osteomyelitis. Patients should be evaluated for a past diagnosis of diabetes, peripheral vascular disease, immunodeficiency, osteomyelitis, recent infections, trauma, animal bites, or surgeries. Further inquiry into social history should also be made, as intravenous drug abuse, tobacco usage, and occupational/environmental exposure also play a role in the pathogenesis of the infection. A thorough history and physical examination often reveals the most likely etiology of an infection. Work-up of osteomyelitis involves laboratory assessment and radiographic investigation. Routine tests include but are not limited to: complete blood count, inflammatory/infectious markers (CRP, ESR, Procalcitonin), and local tissue or surgical bone cultures. It should be emphasized that although this paper will discuss the radiological modalities employed to diagnosis osteomyelitis, open biopsy and culture remain the gold standard for diagnosis. Additionally, none of the radiologic tests described have the ability to identify the specific pathogen involved, meaning they are not useful for targeting antimicrobial therapy.

\section{X-rays (Plain Films)}

In many patients, $\mathrm{x}$-rays are the first diagnostic tool utilized in the radiographic work-up of osteomyelitis. X-rays are widely available and inexpensive; however, they are limited in their ability to detect osteolytic changes. Early radiographic findings may include: soft tissue or deep soft tissue swelling, muscle swelling, or blurred soft tissue planes. ${ }^{5}$ Early bone findings may include: periosteal thickening, lytic lesions, endosteal scalloping, osteopenia, loss of trabecular architecture, and new bone apposition. ${ }^{6}$ Pineda et al. reports that osteomyelitis must extend at least $1 \mathrm{~cm}$ and compromise 30 to $50 \%$ of bone mineral content to produce visible changes in plain radiographs. ${ }^{5}$ Pineda reports the sensitivity and specificity of plain films to be $43-75 \%$ and $75-83 \%$ respectively (Table 1). ${ }^{5}$ A major limitation is that these findings may not be present for 10-21 days after the onset of an infection (Table 2). ${ }^{7}$ As such, x-rays may be more useful for patients who have had a delay in seeking care and did not present until greater than three weeks after symptom onset. A strength of x-rays is the ability to detect alternative diagnoses such as metastatic lesions or osteoporotic fractures. ${ }^{8}$ Despite their limitations, $\mathrm{x}$-rays should routinely be utilized in patients with suspected osteomyelitis. They provide an 
inexpensive tool to evaluate for alternative pathology while their sensitivity improves with time from initial presentation. However, if the diagnosis remains unclear after x-rays and laboratory testing, further imaging should be obtained.

\section{Magnetic Resonance Imaging}

Magnetic resonance imaging (MRI) is a vital tool for the initial diagnosis of acute osteomyelitis. MRI has the ability to detect changes in bone marrow within three to five days of infection, offering a distinct advantage over X-rays. ${ }^{5} \mathrm{MRI}$ also has the ability to detect necrotic bone, sinus tracts, and abscesses, and it can be used to formulate preoperative plans and guide surgical debridement. ${ }^{9-11}$ Sinus tracts, fistulas, and abscess visualization can be further enhanced by the use of gadolinium contrast. However, gadolinium based contrast has been linked to nephrogenic systemic fibrosis and should be used with extreme caution or avoided altogether in patients with moderate to severe renal impairment. ${ }^{12}$

In a meta-analysis of 16 studies, MRI was found to be superior to plain films, three phase technetium bone scan, and leukocyte scan for the evaluation of acute osteomyelitis in the setting of diabetic foot ulcers. ${ }^{11}$ Another meta-analysis of 5 studies evaluating the use of MRI in chronic osteomyelitis found that the sensitivity and specificity was $84 \%$ (95\% CI: 69-92) and 60\% (95\% CI: 38-78) respectively (Table 1). ${ }^{13}$ The specificity of MRI is limited by the fact that bone marrow edema is a non-specific finding that can also be caused by problems such as contusion, fracture, arthritis, or neoplasm. The sensitivity and specificity of MRI also depends on the suspected site of infection. For native vertebral osteomyelitis, MRI has been reported to have a sensitivity and specificity of $97 \%$ and $93 \%$ respectively, and is therefore the primary imaging modality recommended by the IDSA. ${ }^{14}$

MRI does have limitations, one of the largest being its relatively high cost and poor availability to some patients (Table 2). Furthermore, metal and electronic implants may pose a contraindication to the exam or limit the study by producing disruptive artifact. ${ }^{5,15}$ Despite these drawbacks, the literature supports the routine use of MRI in the process of diagnosing acute osteomyelitis. 5,13

\section{Bone Scintigraphy}

Bone scintigraphy, commonly referred to as a bone scan, is another imaging option for diagnosing osteomyelitis. Three different scans are routinely employed: three phase bone scan, gallium scintigraphy, and radio-labeled WBC scan. The three phase bone scan utilizes the ${ }^{99 \mathrm{~m}} \mathrm{Tc}-$ diphosphonate radiopharmaceutical. The first phase involves nuclear angiography, obtaining consecutive two to five second images of the suspected bone during the administration of the radiopharmaceutical. The second phase is obtained within five minutes of administration. Inflammation results in capillary dilation which leads to increased blood flow and pooling. The third phase is obtained approximately three hours later. This phase helps to differentiate between diffuse cellulitis and bone involvement. Osteomyelitis classically results in focal uptake during the third phase whereas cellulitis demonstrates either normal or diffuse uptake resulting from regional hyperemia. ${ }^{16}$ These scans have a high sensitivity but are poorly specific; false positives 
can occur in the setting of recent trauma, prosthetic implants, crystal arthropathy, arthritis, diabetes, or neoplasia (Table 2). ${ }^{15,17}$

Gallium scintigraphy utilizes a radiogallium isotope that attaches to the transferrin that leaks from inflamed blood vessels. Inflammation could be due to either infectious or sterile causes. Gallium scans do not show the same level of bony detail as technetium 3-phase scans, limiting their ability to differentiate between bone and soft tissue pathology. ${ }^{5,16}$ Gallium scans are most useful for diagnosing native vertebral osteomyelitis; IDSA guidelines recommend a combined gallium $/{ }^{99 \mathrm{~m}} \mathrm{Tc}$ scan for patients who have a contraindication to MRI. This combination has a sensitivity of $91 \%$ and a specificity $>90 \% .{ }^{14}$

The third method of bone scanning involves indium-111 labeled leukocytes $\left({ }^{99 \mathrm{~m}} \mathrm{Tc}\right.$-hexamethylpropyleneamine oxime in some studies). Similar to a gallium scan, labeled WBC scans provide poor detail of bony structures. Their advantage is having improved specificity compared to the other bone scans; they are especially useful in cases where other conditions are superimposed. ${ }^{5,18}$ Schauwecker et al. reported a sensitivity and specificity of $88 \%$ and $85 \%$ respectively (Table 1). ${ }^{16}$ Therefore, bone scintigraphy represents a valuable option in the diagnostic imaging of osteomyelitis, and may be especially useful in cases where MRI is unavailable.

\section{Computed Tomography}

Computed tomography (CT) has a number of advantages over X-rays; it allows for improved visualization of intramedullary and soft tissue gas, sequestrum, involucrum, sinus tracts, and foreign bodies. ${ }^{5} \mathrm{CT}$ has superior ability to assess bony architecture and detect necrotic bone (sequestrum) when compared to MRI. The number of cuts in CT is generally greater than those of MRI, reducing the likelihood that pathology may be missed due to small size. This advantage is time dependent, as necrosis may take up to six weeks to develop after the onset of infection. ${ }^{3}$ Thus, during the initial stages of the infection, detection of necrotic bone may not be possible. Sequestered bone is strongly suggestive of an infectious etiology. ${ }^{5}$ Additionally, CT can detect changes such as soft tissue edema or bone destruction earlier than X-rays. CT can also be used to guide aspiration and needle biopsies. ${ }^{5}$ Compared to MRI, the sensitivity and specificity of CT is less impressive. In a meta-analysis, Termaat and colleagues report a sensitivity of $67 \%(95 \% \mathrm{CI}$ : 24-94) and a specificity of 50\% (95\% CI: 3-97) (Table 1). ${ }^{13}$ The drawbacks of computed tomography are the increased cost and radiation exposure compared to plain films; studies may also be limited by the presence of metallic implants or foreign bodies (Table 2). ${ }^{18,19}$

Nevertheless, CT should be strongly considered in patients who are unable to undergo MRI.

\section{Positron Emission Tomography}

Another imaging modality less frequently employed is fludeoxyglucose $\left({ }^{18} \mathrm{~F}\right)$ Positron Emission Tomography (PET). The PET scan relies upon the increased expression of glucose transporters in inflammatory cells and measures the uptake of radio-labeled glucose molecules. This technique can produce results within 30 to 60 minutes of tracer administration; it is unaffected by metal implants or foreign bodies, and produces images with higher special resolution than single photon emitting tracers. ${ }^{20}$ This modality has demonstrated superior sensitivity and specificity to MRI, bone scintigraphy, and leukocyte scintigraphy. ${ }^{13,15}$ A meta-analysis of four studies 
revealed a pooled sensitivity of $96 \%$ (95\% CI: 88-99) and a specificity of $91 \%$ (95\% CI: 81-95)

(Table 1). ${ }^{13}$ PET scan has had limited use in clinical practice due to high cost and poor availability; however, in the future it may become more cost effective, as this modality has demonstrated a high level of diagnostic value (Table 2).

\section{Ultrasound}

The use of ultrasound as an imaging modality for osteomyelitis is less discussed in the literature. However, it offers a valuable alternative given the widespread access and relatively low cost. Ultrasound can be performed at the bedside and poses minimal risk to the patient. It can also be used to delineate infectious etiologies from tumors or noninfectious causes. Similar to CT and MRI, ultrasound can guide biopsies or aspirations and to assess the extent of soft tissue involvement. ${ }^{21}$ Findings such as periosteal elevation, hypoechoic fluid collections around bone, and soft tissue abscesses are suggestive of osteomyelitis. ${ }^{5}$ Ultrasound has also been shown to be an exceptional modality for detecting osteomyelitis in sickle-cell patients. ${ }^{22}$ It should be noted that ultrasound may be more reliable in children than adults due to a looser adherence of periosteum to cortex in the immature skeleton (Table 2). ${ }^{5}$ A drawback to this modality is a lack of studies looking at its reliability in the diagnosis of adult osteomyelitis, although one study found a false-positive rate of $10.5 \%(n=19) .{ }^{23}$ At this time, ultrasound may be best used in combination with other imaging modalities or when other options are unavailable, and prudence should be used in its interpretation.

\section{Conclusion}

The authors conclude that plain films are an appropriate first step in imaging for osteomyelitis, as they may reveal osteolytic changes and can help rule out alternative pathology. MRI is often the most appropriate second study, as it is highly sensitive and can detect bone marrow changes within days of an infection (Table 1). Other studies such as CT, ultrasound, and bone scintigraphy may be useful in patients who cannot undergo MRI (Table 2). CT is useful for identifying necrotic bone in chronic infections. Ultrasound may be useful in children or those with sickle-cell disease. Bone scintigraphy is particularly useful for vertebral osteomyelitis. Finally, PET scan has demonstrated high sensitivity and specificity; however, its clinical application is limited by its high cost and poor availability. When used appropriately, diagnostic imaging can provide high sensitivity and specificity for detecting osteomyelitis, making radiological evaluation a crucial step in the diagnostic process of this debilitating condition.

\section{Tables}

Table 1. Overview of the literature regarding diagnostic imaging of osteomyelitis. Diagnostic levels of evidence are listed as described by Wright et al. ${ }^{24}$ 


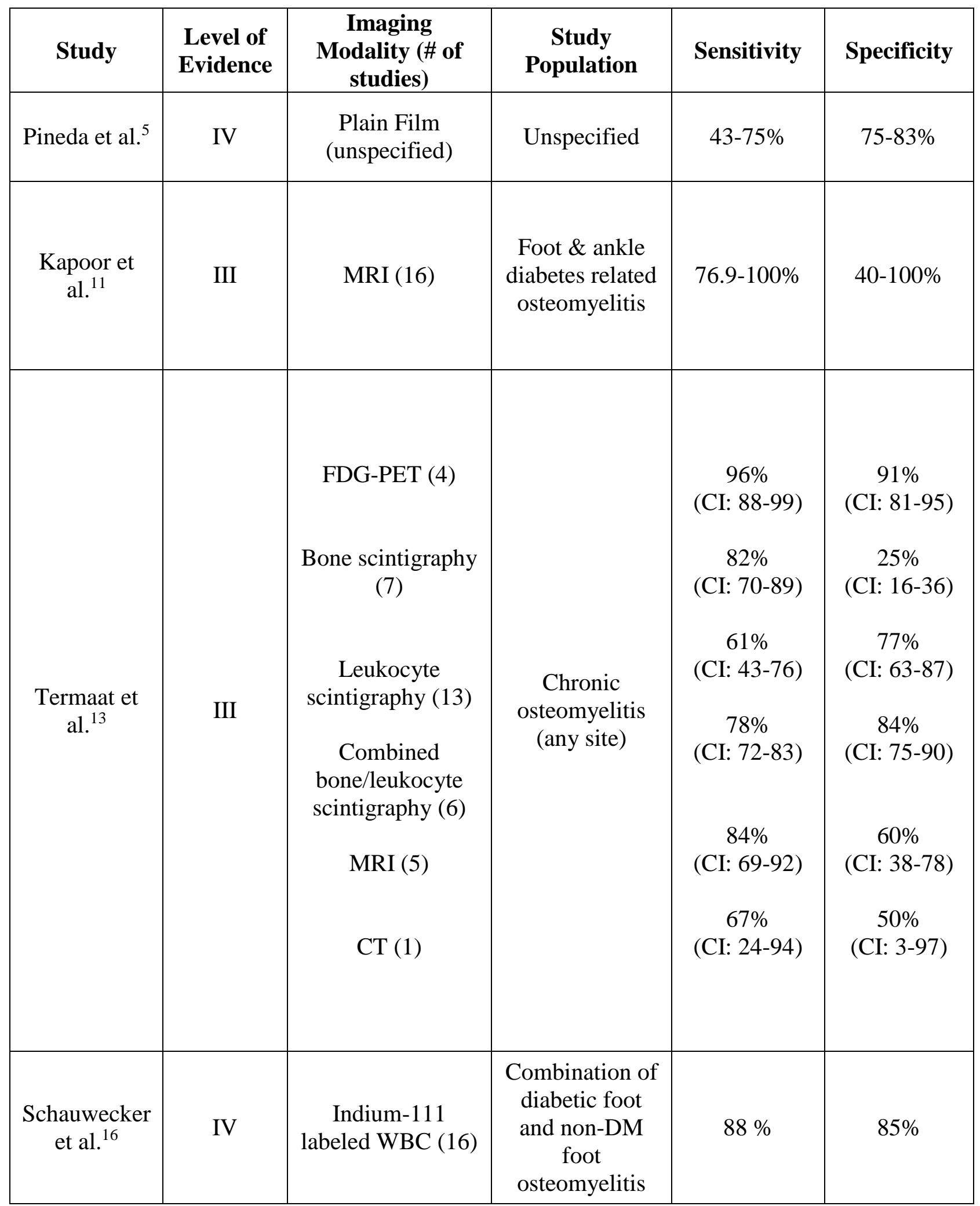


Table 2. Pros, cons, and approximate cost of various imaging modalities.

\begin{tabular}{|c|c|c|c|}
\hline $\begin{array}{c}\text { Imaging } \\
\text { Modality }\end{array}$ & Pros & Cons & $\mathbf{C o s t}^{25,26}$ \\
\hline X-Ray & Cheap & $\begin{array}{c}\text { Low sensitivity, } \\
\text { delayed findings }\end{array}$ & $\sim \$ 50-100$ \\
\hline US & $\begin{array}{c}\text { Cheap, useful in children and sickle cell } \\
\text { patients }\end{array}$ & $\begin{array}{c}\text { Low sensitivity, } \\
\text { difficult interpretation }\end{array}$ & $\sim \$ 100-200$ \\
\hline $\begin{array}{c}\text { Bone } \\
\text { Scintigraphy }\end{array}$ & High sensitivity & Poor specificity & $\sim \$ 150-600$ \\
\hline CT & $\begin{array}{c}\text { Useful for bony architecture, necrotic } \\
\text { bone in chronic osteomyelitis, can guide } \\
\text { biopsy }\end{array}$ & $\begin{array}{c}\text { Increased cost and } \\
\text { radiation exposure }\end{array}$ & $\sim 400-700$ \\
\hline MRI & $\begin{array}{c}\text { Highly specific for both acute and } \\
\text { chronic osteomyelitis }\end{array}$ & High cost & $\sim \$ 700-1200$ \\
\hline PET & Highly specific & $\begin{array}{c}\text { High cost, limited } \\
\text { availability }\end{array}$ & $\sim \$ 1000-2000$ \\
\hline
\end{tabular}




\section{References}

1. Kremers HM, Nwojo ME, Ransom JE, Wood-Wentz CM, Melton LJ, 3rd, Huddleston PM, 3rd. Trends in the epidemiology of osteomyelitis: a population-based study, 1969 to 2009. J Bone Joint Surg Am. 2015;97(10):837-45.

2. Fritz JM, McDonald JR. Osteomyelitis: approach to diagnosis and treatment. Phys Sportsmed. 2008;36(1):nihpa116823-nihpa.

3. Mylona E, Samarkos M, Kakalou E, Fanourgiakis P, Skoutelis A. Pyogenic vertebral osteomyelitis: a systematic review of clinical characteristics. Seminars in arthritis and rheumatism. 2009;39(1):10-7.

4. Concia E, Prandini N, Massari L, Ghisellini F, Consoli V, Menichetti F, et al. Osteomyelitis: clinical update for practical guidelines. Nuclear medicine communications. 2006;27(8):645-60.

5. Pineda C, Espinosa R, Pena A. Radiographic imaging in osteomyelitis: the role of plain radiography, computed tomography, ultrasonography, magnetic resonance imaging, and scintigraphy. Seminars in Plastic Surgery. 2009;23(2):80-9.

6. Kothari NA, Pelchovitz DJ, Meyer JS. Imaging of musculoskeletal infections. Radiologic clinics of North America. 2001;39(4):653-71.

7. Lew DP, Waldvogel FA. Osteomyelitis. Lancet (London, England). 2004;364(9431):369-79.

8. Hatzenbuehler J, Pulling TJ. Diagnosis and management of osteomyelitis. American family physician. 2011;84(9):1027-33.

9. Jevtic V. Vertebral infection. European radiology. 2004;14 Suppl 3:E43-52.

10. Towers JD. The use of intravenous contrast in MRI of extremity infection. Semin Ultrasound CT MR. 1997;18(4):269-75.

11. Kapoor A, Page S, Lavalley M, Gale DR, Felson DT. Magnetic resonance imaging for diagnosing foot osteomyelitis: a meta-analysis. Archives of internal medicine. 2007;167(2):125-32.

12. Wertman R, Altun E, Martin DR, Mitchell DG, Leyendecker JR, O'Malley RB, et al. Risk of nephrogenic systemic fibrosis: evaluation of gadolinium chelate contrast agents at four American universities. Radiology. 2008;248(3):799-806.

13. Termaat MF, Raijmakers PG, Scholten HJ, Bakker FC, Patka P, Haarman HJ. The accuracy of diagnostic imaging for the assessment of chronic osteomyelitis: a systematic review and meta-analysis. J Bone Joint Surg Am. 2005;87(11):2464-71.

14. Berbari EF, Kanj SS, Kowalski TJ, Darouiche RO, Widmer AF, Schmitt SK, et al. 2015 Infectious Diseases Society of America (IDSA) clinical practice guidelines for the diagnosis and treatment of native vertebral osteomyelitis in adults. Clinical infectious diseases : an official publication of the Infectious Diseases Society of America. 2015;61(6):e26-46.

15. Santiago Restrepo C, Gimenez CR, McCarthy K. Imaging of osteomyelitis and musculoskeletal soft tissue infections: current concepts. Rheum. Dis. Clin. North Am. 2003;29(1):89-109.

16. Schauwecker DS. The scintigraphic diagnosis of osteomyelitis. AJR American journal of roentgenology. 1992;158(1):9-18.

17. Littenberg B, Mushlin AI. Technetium bone scanning in the diagnosis of osteomyelitis: a meta-analysis of test performance. Diagnostic Technology Assessment Consortium. Journal of general internal medicine. 1992;7(2):158-64.

18. Bohndorf K. Infection of the appendicular skeleton. European radiology. 2004;14 Suppl 3:E53-63.

19. Cierny G, 3rd, Mader JT, Penninck JJ. A clinical staging system for adult osteomyelitis. Clin. Orthop. Relat. Res. 2003(414):7-24.

20. Stumpe KD, Strobel K. 18F FDG-PET imaging in musculoskeletal infection. The quarterly journal of nuclear medicine and molecular imaging : official publication of the Italian Association of Nuclear Medicine (AIMN) [and] the International Association of Radiopharmacology (IAR), [and] Section of the So. 2006;50(2):131-42.

21. Cardinal E, Bureau NJ, Aubin B, Chhem RK. Role of ultrasound in musculoskeletal infections. Radiologic clinics of North America. 2001;39(2):191-201.

22. Booz MM, Hariharan V, Aradi AJ, Malki AA. The value of ultrasound and aspiration in differentiating vaso-occlusive crisis and osteomyelitis in sickle cell disease patients. Clinical radiology. 1999;54(10):6369. 
23. Larcos G, Antico VF, Cormick W, Gruenewald SM, Farlow DC. How useful is ultrasonography in suspected acute osteomyelitis? Journal of ultrasound in medicine : official journal of the American Institute of Ultrasound in Medicine. 1994;13(9):707-9.

24. Wright JG, Swiontkowski MF, Heckman JD. Introducing levels of evidence to the journal. The Journal of Bone \& Joint Surgery. 2003;85(1):1-3.

25. Buzzle. Bone Scan Cost [cited 2016 4/25]. Available from: http://www.buzzle.com/articles/bone-scancost.html.

26. Oakwood. Radiology Actual Pricing [cited 2016 4/25]. Available from: https://www.oakwood.org/?id=1561\&sid=9\&ChargeType=Radiology. 